\title{
Research on Quality Resources Sharing Platform Based on Cloud Computing
}

\author{
Wenbo Chu *
}

Liaoning Jianzhu Vocational University, Liaoyang, China

Keywords: Quality Resources; Cloud Computing; Sharing Platform; Sharing Standard.

\begin{abstract}
Cloud computing as a novel technique which aims to analyze and operate big data and complex computing is playing a significant role in daily life. One important function it holds is the ability of sharing resources that include infrastructures, software, applications, and business processes. Virtualization is a core technology for enabling cloud resource sharing. However, most of the existing cloud computing platform not formally adopted Service Oriented Architecture they will be more flexible, extensible, reusable. By using the power of SOA and virtualization in the context of Cloud Computing ecosystem, this paper presents architectural principles and derives interconnected architectural modules to form a reusable and customizable Cloud Computing Open Architecture. We conduct case study to deeply analyze the business and chief principle value of services provided by the Internet. We also present some potential value added services on resource sharing based on our proposed cloud computing platform.
\end{abstract}

\section{Introduction}

As a key service delivery platform in the field of service computing [1], Cloud Computing provides environments to enable resource sharing in terms of scalable infrastructures, middleware and application development platforms, and value-added business applications. Mode of operation may include PAYG utility model, and worthless infrastructure services value-added services platform, value-added application service fees infrastructure services, or free service providers from the consumer and revenue sharing. As the summary in [1], there are usually four types can be configured and consumption in the Internet resources. They can be shared among users take advantage of economies of scale. Provided is a shared resource requests on the network. A major goal of cloud computing is to use the Internet or intranets to provide resources to users. The first type of resources is infrastructure resources, which include computing power, storage, and machine provisioning. For example, Amazon EC2 provides web service interface to easily request and configure capacity online [2]. The second type of resources in Cloud Computing is software resources including middleware and development resources. Understanding the design platform, the development of resource development tools, testing tools, deployment tools, open source based reference project. The third type of resources in Cloud Computing is application resources. The fourth type of resources in Cloud Computing is business processes.

Currently, there is no standard definition or specification for Cloud Computing. It may take some time to define the key characteristics of Cloud Computing based on practices in the field. Cloud computing is a set of key technology to solve the resource sharing based on business needs. According to the design of our practices in the areas of services and solutions, we consider the following two key enabling technologies, can play a very important role in the stage of the Revolution: the system structure of virtual technology and service oriented. Creating a so-called Cloud Computing platform is easy as long as it can enable sharing of at least one of the resources. However, building a unified, scalable and reusable Cloud Computing architecture to support sharing of all types of resources still faces challenges in the areas of technology breakthrough and best industry practices. 


\section{Our Proposed Model}

\section{OSI Model for Cloud Computing.}

We have identified the following three objectives to help address the above challenge of defining a good open architecture for Cloud Computing. The first goal is to elucidate a reusable way to create extensible and configurable computing platform configuration of cloud. This combination of service oriented architecture and virtual power delivery business and new application software, hardware and practical value, and the business process configuration of the network service in the context of cloud computing. The second objective is to propose a set of common construction of cloud computing services platform, to provide business services or other cloud services in a unified way for business users. The third objective is to maximize the potential business value of Cloud Computing based on an extensible IT infrastructure and management system. This will cause the value-added service cloud, joint power SOA and cloud computing through monetization. As we know, OSI standards for Open System Interface [3] which has encountered some challenges to realize its value in the context of generic open systems.

\section{Principles of Cloud Computing Architecture.}

In this Cloud Computing Open Architecture, we propose an integrated co-innovation and coproduction framework to get cloud vendors, cloud partners, and cloud clients to work together based on seven principles, which are used to define ten major architectural modules and their relationships shown in Figure 1. The crucial principles are discussed as follows. (1) Integrated Ecosystem Management for Cloud. The architecture must support the management of the ecosystem of Cloud Computing. This ecosystem includes all involved services and solutions vendors, partners, and end users to provide or consumer shared resources in the Cloud Computing environment. Cloud vendors expose its interaction interfaces of its internal operations and product development capability to the cloud. Cloud partners provide components to cloud vendors or serve as agents to provide value-added services to the cloud clients. Cloud clients are users of the cloud services that offer business goal driven resource sharing. (2) Virtualization for Cloud Infrastructure. There are two basic approaches for enabling virtualization in the Cloud Computing environment. The first method is the hardware virtualization, management plugin hardware equipment and the play mode. Hardware can be added or removed without affecting the normal operation of other equipment in the system. Of course, performance or storage space may be due to the dynamic changes that add and delete operations. The second approach is software virtualization, i.e., to use software image management or software code virtualization technology to enable software sharing. (3) Service-Orientation for Common Reusable Services. Introduced before, in addition to the characteristics of the service oriented virtualization, is another driving force of cloud computing for the further implementation of assets from the reusability, the commercial value of the composite application, and mix of services. Two main types of common reusable services: cloud horizontal and vertical business services. (4) Extensible Provisioning and Subscription for Cloud. Extensible service provisioning is the unique feature of a Cloud Computing system. No scalability, the configuration part of the cloud computing architecture to support only a specific type of resource sharing. This means that the architecture of free use service users and subscribers are all the same. Both types of users can be service providers or consumers from time to time. From service consumers' perspective, they are interested in how to easily access services based on their own business logics and goals. From service providers' perspective, three levels of service provisioning described previously will be the target offerings. (5) Configurable Enablement for Cloud Offerings. Cloud offerings are the final products or services that are provisioned by the Cloud Computing platform. In the Cloud Computing environment, business process as a service is a new model for sharing best practices and business processes among cloud clients and partners in the value chain. An example of the business process as the cloud computing environment provides a service is software testing, which is a software development procedure. It is very important for the business process. Because the software system or application need to deploy and run on different operating system, middleware environment, or different versions or configuration, it is very difficult for individual developers or companies to create a test "factory" solve the problem of testing in the 
application service area. Upgrading or maintaining testing environment involves the migration of hardware, software, and testing knowledge repositories. They are the two major expenses in addition to the testing engineers who manipulate the environment to conduct the testing. (6) Unified Information Representation and Exchange Framework. Information representation and message exchange of Cloud Computing resources are very important to enable the collaborative and effective features of Cloud Computing. (7) Cloud Quality and Governance. The last and most important module in out platform is the Cloud Quality and Governance shown in Figure 1. This module is responsible for the identification and definition of quality indicators for Cloud Computing environment and a set of normative guidance to govern the design, deployment, operation, and management of the cloud offerings.

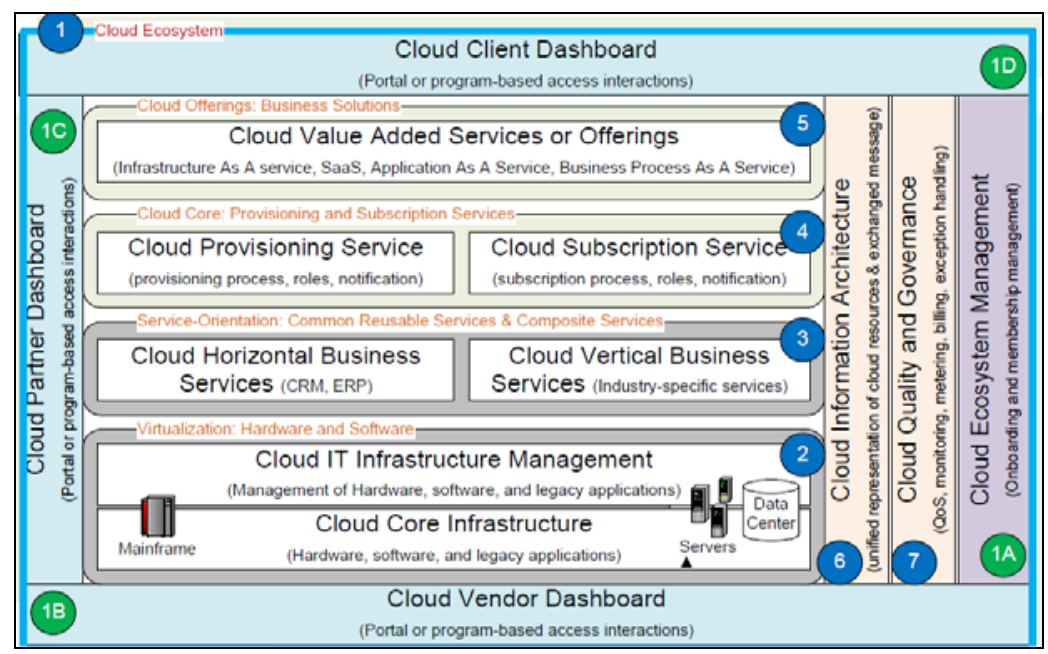

Figure. 1 The Cloud Computing Open Architecture Overview Diagram

\section{Experiment Analysis and Case Studies}

In this case study, we build our own private cloud to enable computing resource sharing. The usage scenario is to offer service requesters a pay-to-use model to provide servers with a selected set of preinstalled software packages to shorten time to production based on request submissions. In the governance aspect, organizations like the Center of Excellence on Cloud Computing and Cloud Operations Team are created to support its overall strategic planning, architecture design and review, alignment, and coordination. The overall productivity, reusability, cost-effectiveness are also identified and reflected in the design of the Cloud solution architecture based on CCOA's seven principles. For example, some policies on when to use Web services and where to allocate machines are also part of the governance process. In the virtualization module, the Cloud Core Infrastructure includes a large number of servers, data centers, and supporting software packages such as WebSphere Application Server (WAS), DB2 database, and LDAP. In this case study, we use WebSphere Process Server to support BPEL processes and WebSphere Portal Server to support portlets. LDAP is used to manage users and access control information. Cloud Infrastructure Management is enabled by Tivoli Enterprise Portal Server and Tivoli Provisioning Manager, to handle the hardware virtualization and provisioning in this Infrastructure Cloud. As an example, we use the Cloud Vendor Dashboard to monitor the usages and resource statistics which are granted by the Cloud Quality and Governance module. Figure 2 illustrates the statistic data chart of the Cloud Computing resources. This usage diagram shows how many resources are available for cloud clients to subscribe. The resources are categorized based on server types and operating systems (e.g. Windows and Linux). In this case, there are Xen-VM, xSeries, Xen Cluster, VMware, DynamicP6, and DynamicP. We have two operating systems (Windows and Linux) for provisioning. As shown in Figure 3, there are one subscribed Xen-VM machine with Linux and one Xen-VM for subscription. There are 17 xSeries for subscription. There are 13 subscribed Xen Cluster machines with Linux and 9 machines for subscription. There are one subscribed VMware machine with 
Windows and one VMware machine for subscription. There are 1 subscribed DynamicP6 with Linux and 5 machines for subscription. There are 4 Dynamic P servers for cloud clients to subscribe [4].

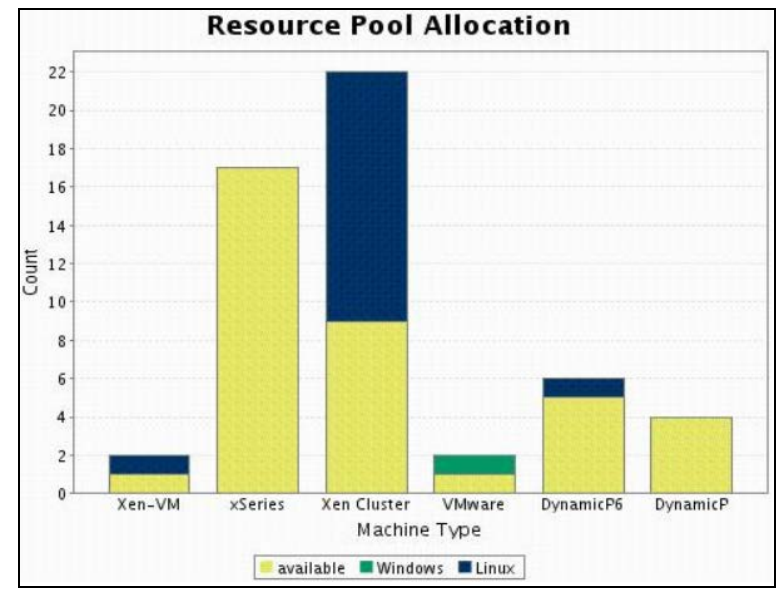

Figure. 2 The Cloud Resource Statistics

\section{Summary and Conclusion}

In this paper, we have proposed the Cloud Computing Open Architecture (CCOA) based on seven architectural principles and ten architectural modules, by integrating the power of service-oriented architecture (SOA) and virtualization technology of hardware and software. The aim of the proposed method is to SOA and virtual force together to help the understanding and discussion of cloud computing business value. We hope to encourage the research community to work together to improve the CCOA and focus on building a solid foundation of cloud computing in CCOA theory. For future research, we will be in the mode of building blocks with and interact in all ten modules of the definition of CCOA, as well as the standard service interface (or API) solution oriented building blocks and managed cloud infrastructure between. We have also presented an Infrastructure Cloud as a case study to illustrate how to use CCOA to enable infrastructure-level resource sharing as a cloud offering. We have also studied a business cloud to illustrate how to use CCOA to separate the concern of business design from infrastructure enablement through Cloud Publishing Business. The case studies and analysis have shown that the proposed CCOA is an extensible and configurable architecture for providing normative guidance and enabling infrastructure, software, application, and business process sharing in a unified manner.

\section{References}

[1] Liang-Jie Zhang, Qun Zhou, Jen-Yao Chung, Developing Grid Computing Applications, IBM developer Works Journal, May 2003, pp.10-15.

[2] Jing Min Xu, Ying Nan Zuo, Shun Xiang Yang, Zhong Tian, Henry Chang, Liang-Jie Zhang, Tian Chao: Membership Portal and Service Provisioning System for an Infrastructure of Hubs: Managed EHub. ICEIS (4) 2003: pp. 143-150.

[3] Liang-Jie Zhang, Haifei Li, Herman Lam, Toward a Business Process Grid for Utility Computing, IT Professional, Volume: 6, Issue: 5, Sept.-Oct. 2004, Pages: 64 - 63.

[4] Liang-Jie Zhang, Jia Zhang, Hong Cai, Services Computing, Springer and Tsinghua University Press, 2007, ISBN: 978-3-540-38281-2, July 2007. 